\title{
Low-frequency pulsed electromagnetic field inhibits RANKL-induced osteoclastic differentiation in RAW264.7 cells by scavenging reactive oxygen species
}

\author{
YING PI $^{1}$, HAIFENG LIANG ${ }^{2}$, QIANG YU ${ }^{2}$, YUKUN YIN ${ }^{2}$, HAIXIA XU ${ }^{2}$, \\ YUTIAN LEI ${ }^{2}$, ZHONGYU HAN $^{2}$ and JING TIAN ${ }^{1}$ \\ ${ }^{1}$ Department of Orthopedics, Zhujiang Hospital, Southern Medical University, Guangzhou, \\ Guangdong 510280; ${ }^{2}$ Department of Human Anatomy, Basic Medical College, \\ Southern Medical University, Guangzhou, Guangdong 510515, P.R. China
}

Received September 11, 2018; Accepted February 15, 2019

DOI: $10.3892 / \mathrm{mmr} .2019 .10079$

\begin{abstract}
Bone homeostasis is a dynamic balance maintained by bone formation and resorption. An increase in the number and activity of osteoclasts leads to excessive bone resorption, which in turn results in bone disease, including osteoporosis. Therefore, inhibiting the differentiation and activity of osteoclasts is important for maintaining bone mass. Several studies have revealed that the use of a low-frequency pulsed electromagnetic field (PEMF) is an effective method to treat osteoporosis. However, its exact mechanism remains to be fully clarified. Therefore, the present study was designed to examine the effects that PEMF exerts on receptor activator of nuclear factor- $\kappa \mathrm{B}$ ligand (RANKL)-induced osteoclastogenesis and intracellular reactive oxygen species (ROS) production in RAW264.7 cells. The viability of cells was determined using a Cell Counting Kit- 8 assay, and gene and protein expression were investigated via reverse transcription-quantitative polymerase chain reaction and western blot analyses. Furthermore, microscopy was performed to detect the levels of intracellular ROS and tartrate-resistant acid phosphatase (TRAP). Following the culture of RAW264.7 cells with RANKL $(50 \mathrm{ng} / \mathrm{ml}$ ) for 4 days ( $3 \mathrm{~h} /$ day) under PEMF ( $75 \mathrm{~Hz}, 1 \mathrm{mt}$ ) exposure, it was observed that PEMF had an inhibitory effect on RANKL-induced osteoclastic differentiation. Multinucleated osteoclast formation, the activity of TRAP and the expression of osteoclastogenesis-associated genes, including cathepsin K, nuclear factor of activated T cells cytoplasmic 1 and TRAP, were significantly reduced by PEMF. Furthermore, PEMF
\end{abstract}

Correspondence to: Professor Jing Tian, Department of Orthopedics, Zhujiang Hospital, Southern Medical University, 253 Industrial Avenue, Haizhu, Guangzhou, Guangdong 510280, P.R. China

E-mail: tianjing_ortho@163.com

Key words: pulsed electromagnetic field, osteoclast, differentiation, reactive oxygen species, receptor activator of nuclear factor- $\kappa \mathrm{B}$ signaling effectively decreased the generation of intracellular ROS during osteoclastic differentiation. In addition, the results demonstrated that ROS are the key factor in osteoclast differentiation and formation. Reducing intracellular ROS with diphenylene-iodonium chloride significantly inhibited RANKL-induced osteoclast differentiation. Taken together, the results of the present study demonstrated that PEMF may inhibit RANKL-induced osteoclastogenesis by scavenging intracellular ROS. These results may provide the groundwork for future PEMF clinical applications in osteoclast-associated bone disease.

\section{Introduction}

It is well known that bone homeostasis is a dynamic balance maintained by bone formation and bone resorption $(1,2)$. Osteoclasts are derived from hematopoietic precursors and function in bone resorption. The classic receptor activator of nuclear factor- $\mathrm{\kappa B}$ (RANK)/RANK ligand (RANKL)/osteoprotegerin signaling pathway regulates the activity and differentiation of osteoclasts. RANKL is considered to be an important factor that regulates osteoclast differentiation and bone metabolism (3). Excessive bone resorption is closely associated with the formation of osteoclasts, which can lead to bone disease, including osteoporosis (4). It has been shown that osteoporosis significantly increases the risk of fracture, particularly hip fractures, in postmenopausal women (5). Therefore, regulating osteoclast differentiation and activity is important for preserving bone mass and reducing the incidence of fractures. At present, the treatment of osteoporosis predominantly includes estrogen replacement therapy and medical treatment, including the use of calcitriol and caltrate D; however, clinical evidence has demonstrated that these treatments are associated with serious side effects, including cancer $(6,7)$. Therefore, identifying novel treatments is critical.

As a non-invasive and inexpensive method, low-frequency pulsed electromagnetic fields (PEMF) have shown efficacy for a wide range of diseases of the skeletomuscular system $(8,9)$. In previous years, it has been reported that PEMF may 
inhibit osteoclast differentiation in vitro $(10,11)$, however, the underlying mechanisms remain to be fully elucidated. PEMF exposure is likely to produce satisfying therapeutic effects for certain bone diseases, including osteoporosis, which is closely associated with osteoclast function $(4,12,13)$. Therefore, in vitro studies examining the effects of PEMF on osteoclasts and the potential underlying mechanisms are essential to understand the efficacy of PEMF for treating osteoclast-associated diseases.

Reactive oxygen species (ROS) are produced intracellularly as byproducts during mitochondrial electron transport or through the action of certain enzymes, including NADPH oxidase and cyclooxygenase (14). ROS consist of radical and non-radical oxygen species, including the superoxide anion $\left(\mathrm{O}_{2-}\right)$, hydroxyl radical $\left(\mathrm{OH}^{-}\right)$and hydrogen peroxide $\left(\mathrm{H}_{2} \mathrm{O}_{2}\right)(15)$. Changes in ROS levels are implicated in regulating cellular signal transduction. The excessive production of ROS results in oxidative stress, which in turn may cause apoptosis, ischemia and inflammation (16). However, low, non-toxic levels of ROS may act as secondary messengers in several receptor signaling pathways (17-19). Various studies have shown that ROS are implicated in bone metabolism and promote osteoclast differentiation and bone resorption (20-23). The increased production of ROS during osteoclast formation appears to activate the peroxisome prol iferator-activated-receptor- $\gamma$ coactivator $1 \beta$, which regulates mitochondrial biogenesis, thus facilitating osteoclast differentiation (24). A previous study illustrated the destructive effects of antioxidants on osteoclastogenesis in mouse models lacking the forkhead box $\mathrm{O}$ transcription factor, which drives transcription of the antioxidant catalase (25). To the best of our knowledge, the inhibitory effects of PEMF on RANKL-induced osteoclast differentiation have not been investigated. In the present study, the effects of PEMF on osteoclast differentiation were examined. It was demonstrated that PEMF served as a RANKL-mediated inhibitor of osteoclastogenesis. The mechanism underlying this inhibitory effect may be via the suppression of ROS generation, which is required for osteoclast differentiation. Therefore, the results of the present study demonstrated that PEMF may inhibit osteoclast differentiation by scavenging intracellular ROS.

\section{Materials and methods}

Cell culture and PEMF exposure. RAW264.7 cells were purchased from the Cell Bank of The Type Culture Collection of Chinese Academy of Sciences (Shanghai, China). For the osteoclastogenesis experiment, the cells were cultured in 24-well culture plate at $1 \times 10^{6}$ cells $/ \mathrm{ml}$, followed by culture in $\alpha$-minimum essential media ( $\alpha$-MEM; Gibco; Thermo Fisher Scientific, Inc., Waltham, MA, USA) containing $10 \%$ fetal bovine serum (FBS, Gibco; The Fisher Scientific, Inc.) and $1 \%$ penicillin $/ 1 \%$ streptomycin in the presence of $50 \mathrm{ng} / \mathrm{ml}$ RANKL (PeproTech, Inc., Rocky Hill, NJ, USA). The medium was placed at $37^{\circ} \mathrm{C}$ in a humidified atmosphere containing $5 \% \mathrm{CO}_{2}$ for 4 days and refreshed every $48 \mathrm{~h}$.

For PEMF, the cells were placed in an incubator containing a $75 \mathrm{~Hz}$ sinusoidal PEMF with a density of $1 \mathrm{mT}$. The magnetic field used for the test was produced via a Helmholtz coil. The cells in the control group were placed in another incubator under the same conditions, but without a PEMF in the incubator.

Cell Counting Kit-8 (CCK-8) proliferation assay. Cell viability was quantified according to the manufacturer's protocol using the CCK-8 kit (Dojindo Molecular Technologies, Inc., Kumamoto, Japan). Briefly, the RAW264.7 cells were seeded in 96-well plates at a cell density of 1,000 cells/well and cell proliferation was determined at different time points (each group had a set of three sub-wells). The PEMF group was exposed to PEMF for 4 days ( $3 \mathrm{~h} /$ day), whereas control cells were cultured under the same conditions without PEMF. After 24, 48, 72 and 96 h, $10 \mu$ l CCK-8 solution was added to each well. Following incubation at $37^{\circ} \mathrm{C}$ for $4 \mathrm{~h}$, the optical density (OD) of each well was determined using an enzyme mark instrument (Multiskan FC; Thermo Fisher Scientific, Inc.) at a wavelength of $450 \mathrm{~nm}$.

Tartrate-resistant acid phosphatase (TRAP) staining, cell counting andTRAP activity assay. Following exposure to PEMF, the cells were washed with PBS and fixed. A TRAP staining kit (Sigma-Aldrich; Merck KGaA, Darmstadt, Germany) was used to identify the formation of mature osteoclasts according to the manufacturer's protocol. The cell culture plate was transferred to an optical microscope, and cells that were TRAP-positive with more than three nuclei were confirmed to be osteoclasts. Five random fields of view were selected, and the number of osteoclasts was counted. TRAP activity was measured at a wavelength of $540 \mathrm{~nm}$ with a microplate reader.

Determination of intracellular ROS. The level of intracellular ROS generated by $\mathrm{H}_{2} \mathrm{O}_{2}$ was measured using a ROS assay kit (Beyotime Institute of Biotechnology, Haimen, China). $\mathrm{H}_{2} \mathrm{O}_{2}$ was used as a ROS inducer. DCFH-DA interacts with ROS in viable cells to generate 2',7'-dichlorofluorescein (DCF). DCF is highly fluorescent at $530 \mathrm{~nm}$. The cells were washed with PBS three times and cultured for $30 \mathrm{~min}$ at $37^{\circ} \mathrm{C}$ in the dark following the addition of DCFH-DA at a final concentration of $10 \mu \mathrm{M} / \mathrm{ml}$. The relative expression of ROS was evaluated using a fluorescent microscope, and the fluorescence intensity of DCF was determined using a fluorometric plate reader. To further investigate the involvement of ROS in the promotion of osteoclast precursor differentiation, diphenylene-iodonium chloride (DPI; Sigma-Aldrich; Merck KGaA), a widely used NADH oxidase 1 inhibitor that completely scavenges generated ROS, was added to the cultured system (RANKL).

Reverse transcription-quantitative polymerase chain reaction $(R T-q P C R)$ analysis. TRIzol ${ }^{\circledR}$ reagent (Thermo Fisher Scientific, Inc.) was used to extract intracellular total RNA, and a Prime Script RT kit (Takara Bio, Inc., Otsu, Japan) was used to synthesize single-stranded cDNA. qPCR was performed using an ABI 7500 real-time PCR system (Thermo Fisher Scientific, Inc.). qPCR was conducted using cDNA (1 $\mu \mathrm{l})$ with SYBR Green-1 (20 $\mu \mathrm{l}$; Takara Bio, Inc.) according to the manufacturer's protocol under the following conditions: Activation at $95^{\circ} \mathrm{C}$ for $10 \mathrm{~min}$, then 40 cycles of amplification $\left(95^{\circ} \mathrm{C}\right.$ for $10 \mathrm{sec}, 60^{\circ} \mathrm{C}$ for $24 \mathrm{sec}$ and $72^{\circ} \mathrm{C}$ for $20 \mathrm{sec}$ ) and a final extension at $72^{\circ} \mathrm{C}$ for $1 \mathrm{~min}$. All the reactions were performed in triplicate and target gene expression 
A

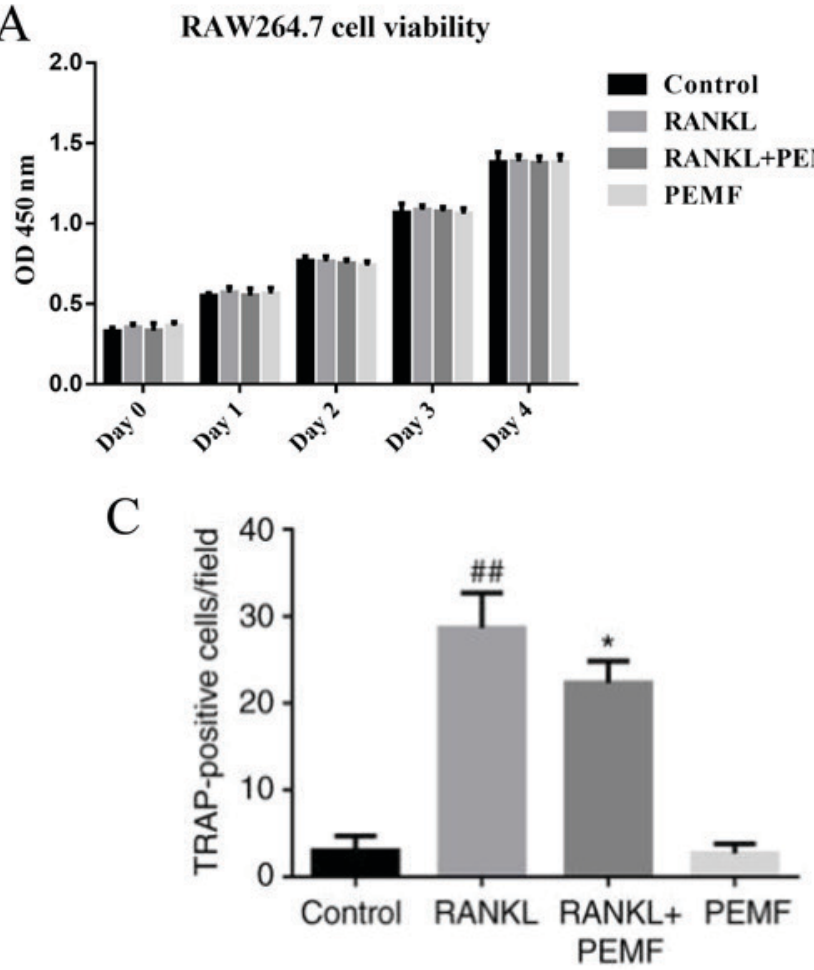

B

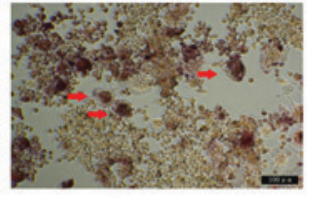

Control

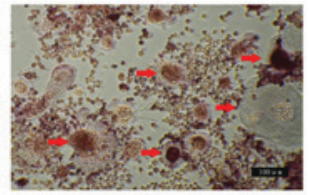

RANKL+PEMF

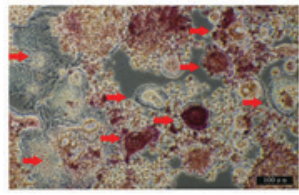

RANKL

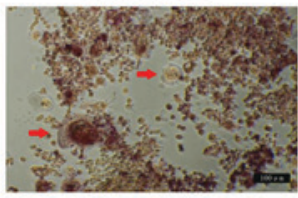

PEMF
D

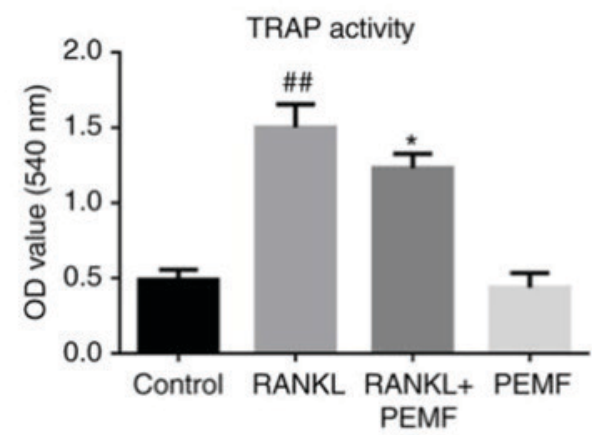

Figure 1. PEMF inhibits osteoclastic differentiation, but not the viability of RANKL-induced RAW264.7 cells. Cells were treated with or without RANKL (50 ng/l) under PEMF stimulation for 4 days. (A) Cell viability was analyzed using the Cell Counting Kit- 8 assay. (B) After 4 days in culture, cells were fixed and stained using the TRAP kit. Osteoclasts were visualized under an optical microscope. Scale bar $=100 \mu \mathrm{m}$. Red arrows indicate multinucleated osteoclasts. (C) TRAP-positive multinucleated osteoclasts containing more than three nuclei were counted in five random fields. (D) TRAP activity was measured at $\lambda=540 \mathrm{~nm}$. Values are expressed as the mean \pm standard deviation. $(\mathrm{n}=3) .{ }^{\# \#} \mathrm{P}<0.01$ compared with the control group; ${ }^{*} \mathrm{P}<0.05$ compared with $\mathrm{RANKL}$ alone. PEMF, pulsed electromagnetic field; RANKL, receptor activator of nuclear factor- $\kappa$ B ligand; TRAP, tartrate-resistant acid phosphatase; OD, optical density.

was normalized to the reference gene $\beta$-actin. The $2^{-\Delta \Delta C q}$ method (26) was applied to calculate the relative expression level of the target genes. The PCR products were subjected to melting curve analysis and a standard curve to confirm the correct amplification. The primers used for PCR were as follows: Cathepsin K (CTSK), forward 5'-AGAACGGAG GCATTGACTCT-3', reverse 5'-GATGGACACAGAGATGGG TC-3'; TRAP, forward 5'-CGATCACAATCTGCAGTACC-3', reverse 5'-ACCCAGTGAGTCTTCAGTCC-3'; nuclear factor of activated T cells cytoplasmic 1 (NFATc1), forward 5'-CGCAAG TACAGTCTCAATGG-3', reverse 5'-CAGGTATCTTCGGTC ACACT-3'; and $\beta$-actin, forward 5'-AGGCCAACCGTGAAA AGATG-3' and reverse 5'-TGGCGTGAGCGAGACCATAG-3'.

Western blotting. The treated RAW264.7 cells $\left(5 \times 10^{4}\right.$ cells per well) were lysed using cOmplete ${ }^{\mathrm{TM}}$ Lysis-M and Phos-STOP (Roche Diagnostics, Indianapolis, IN, USA). The concentration was measured using a Bicinchoninic Acid protein assay kit (Pierce; Thermo Fisher Scientific, Inc.), Proteins (40 $\mu \mathrm{g} / \mathrm{lane})$ were separated via $10 \%$ SDS-PAGE. The proteins were then transferred to polyvinylidene difluoride (PVDF) membranes, and were blocked for $1.5 \mathrm{~h}$ at room temperature with $3 \%$ non-fat dry milk in TBS-Tween-20 (150 mM NaCl, pH 7.4, $25 \mathrm{mM}$ Tris- $\mathrm{HCl}$ and $0.2 \%$ Tween-20). The membranes were incubated with specific primary antibodies overnight at $4^{\circ} \mathrm{C}$. The primary rabbit polyclonal antibodies used were as follows: Anti- $\beta$-actin (1:1,000; cat. no. ab8226, Abcam, Cambridge, MA, USA); anti-CTSK (1:2,000; cat. no. 11239-1-AP, ProteinTech Group,
Inc., Chicago, IL, USA); anti-NFATc1 (1:3,000; cat. no. 4389, Cell Signaling Technology, Inc., Danvers, MA, USA) and anti-TRAP (1:2,000; cat. no. 10325-1-AP, ProteinTech Group, Inc.). Membranes were then incubated for $1 \mathrm{~h}$ at room temperature with horseradish peroxidase-conjugated goat anti-rabbit immunoglobulin G secondary antibodies (1:1,000; cat. no. 7074, Cell Signaling Technology, Inc.). Immunoreactive bands on the PVDF membranes were detected by enhanced chemiluminescence (Tanon Science and Technology Co., Ltd., Shanghai, China).

Statistical analysis. Data are presented as the mean \pm standard deviation. Each experiment was repeated at least three times. Statistical analyses were performed using one-way analysis of variance with subsequent post hoc multiple comparisons with Dunnett's test using SPSS version 20 (IBM Corp., Armonk, $\mathrm{NY}$, USA). $\mathrm{P}<0.05$ was considered to indicate a statistically significant difference.

\section{Results}

PEMF inhibits the osteoclastic differentiation, but not the viability of $R A N K L$-induced $R A W 264.7$ cells. Cell viability was assessed using a CCK- 8 assay following exposure of the RAW264.7 cells to PEMF. The RAW264.7 cells were treated with or without RANKL (50 ng/l) for 4 days under PEMF $(75 \mathrm{~Hz}, 1 \mathrm{mt}, 3 \mathrm{~h} /$ day). The results revealed no significant difference in cell viability between groups (Fig. 1A). 

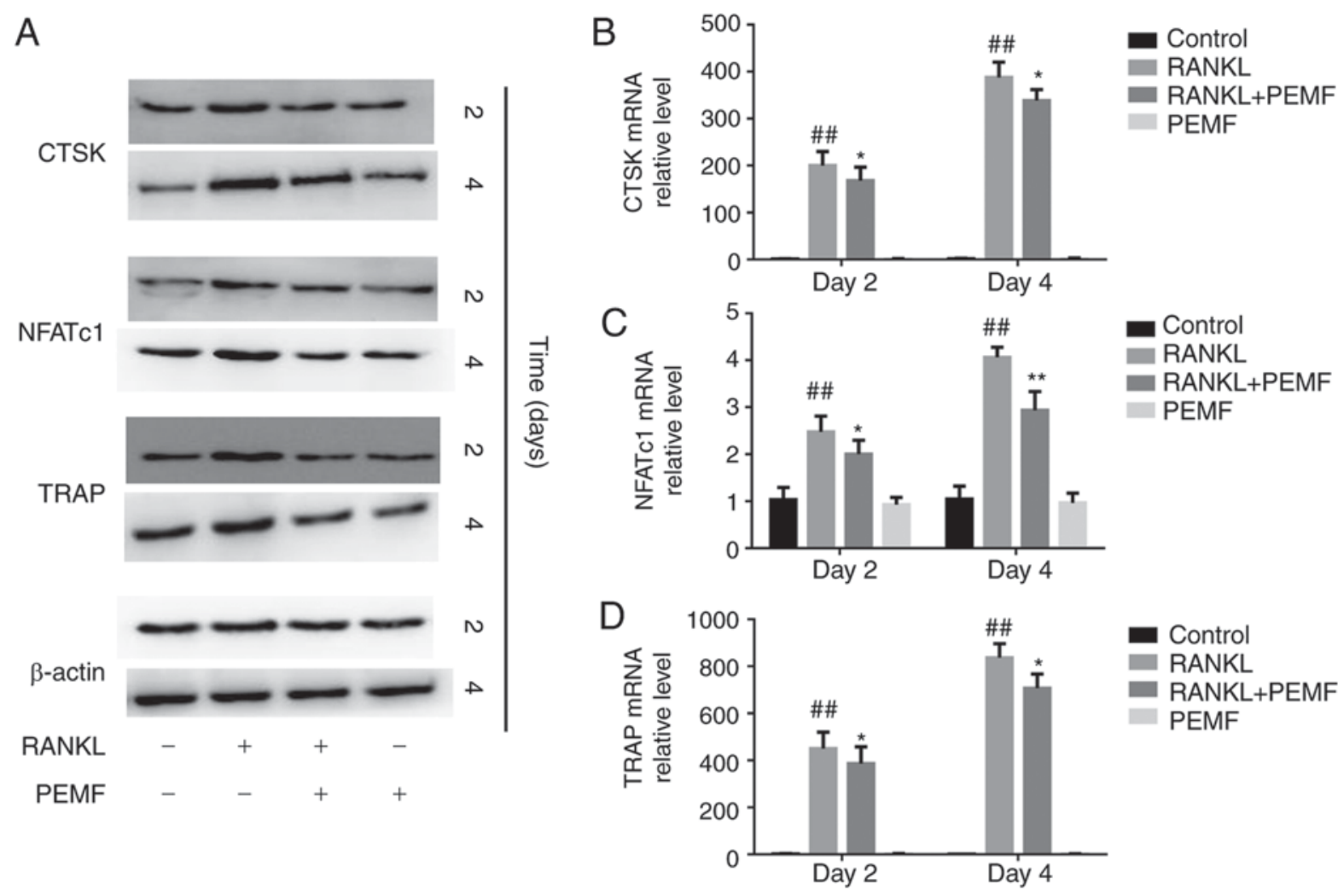

Figure 2. PEMF inhibits the expression of osteoclastogenesis-associated genes. RAW264.7 cells were cultured with RANKL (50 ng/ml) for 2 or 4 days with PEMF exposure. (A) Expression of osteoclastogenesis-related genes (CTSK, NFATc1 and TRAP) was determined by western blotting. Expression levels of osteoclastogenesis-(B) CTSK, (C) NFATc1 and (D) TRAP were examined by reverse transcription-quantitative polymerase chain reaction analysis. Values are expressed as the mean \pm standard deviation $(n=3)$. The relative expression level of the target gene was normalized to that of $\beta$-actin. ${ }^{\# \#} \mathrm{P}<0.01$ compared with the control group; ${ }^{*} \mathrm{P}<0.05$ and ${ }^{* *} \mathrm{P}<0.01$ compared with RANKL alone. PEMF, pulsed electromagnetic field; RANKL, receptor activator of nuclear factor- $\kappa \mathrm{B}$ ligand; CTSK, cathepsin K; NFATc1, nuclear factor of activated T cells cytoplasmic 1; TRAP, tartrate-resistant acid phosphatase.

In the present study, the inhibitory effect of PEMF on osteoclast differentiation was investigated. RANKL $(50 \mathrm{ng} / \mathrm{ml})$ induced the differentiation of RAW264.7 murine macrophage cells into osteoclasts. Osteoclasts were visualized under an optical microscope (Fig. 1B). However, PEMF significantly reduced the number of TRAP-positive multinucleated osteoclasts (Fig. 1C). Compared with the control group, RANKL treatment increased the activity of TRAP by $150 \%$, whereas PEMF reduced the activity of TRAP stimulated by RANKL (Fig. 1D). No significant difference was observed between the control group and PEMF group.

PEMF inhibits the expression of osteoclastogenesis-associated genes. The expression of several osteoclastogenesis-associated genes, including CTSK, NFATc1 and TRAP, were determined by western blotting (Fig. 2A) and RT-qPCR analysis (Fig. 2B-D) on days 2 and 4. The results showed that PEMF inhibited the RANKL-induced increase in the expression of CTSK, NFATc1 and TRAP, which was consistent with the above results.

Antioxidant capacity and intracellular ROS levels are reduced by PEMF. To confirm whether PEMF can scavenge intracellular ROS, the accumulation of intracellular ROS was evaluated by fluorescence microscopy. The RAW264.7 cells were exposed to PEMF for $3 \mathrm{~h}$ following treatment with $1 \mathrm{mM}$ $\mathrm{H}_{2} \mathrm{O}_{2}$ for 30 min. A previously reported study demonstrated that during RANKL-induced differentiation of RAW264.7 cells into osteoclasts, maximum intracellular ROS levels were reached at day 2 (27). Therefore, the inhibitory effect of PEMF on ROS production was assessed following 2 days of induction with RANKL $(50 \mathrm{ng} / \mathrm{ml})$. The ROS-positive cells were visualized as bright green spots, and $\mathrm{H}_{2} \mathrm{O}_{2}$ treatment increased the level of intracellular ROS, compared with that in the control group. In addition, treatment with PEMF inhibited the $\mathrm{H}_{2} \mathrm{O}_{2}$-induced increase in intracellular ROS (Fig. 3A). All cells were collected and analyzed on a fluorometric plate reader to measure the OD values of DCF. The fluorescence intensity of DCF was reduced in the PEMF $+\mathrm{H}_{2} \mathrm{O}_{2}$ group compared with that in the $\mathrm{H}_{2} \mathrm{O}_{2}$-treated group (Fig. 3B). In the RAW264.7 cells treated with RANKL and exposed to PEMF for 2 days ( $3 \mathrm{~h} /$ day), it was determined that RANKL treatment significantly increased ROS, and PEMF prevented this increase (Fig. 3C). Similar results were observed for the OD values of DCF (Fig. 3D). These results suggested that PEMF had antioxidant capacity and reduced intracellular ROS.

Reducing intracellular ROS levels inhibits RANKL-induced osteoclast differentiation. The results showed that DPI administration inhibited the effect of RANKL on osteoclast differentiation and reduced the number of TRAP-positive multinuclear cells (Fig. 4A and B). The decreased activity of TRAP (Fig. 4C) was consistent with the observed alterations in the TRAP staining. DPI treatment also 
A

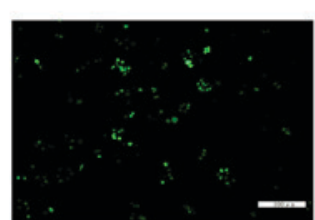

Control

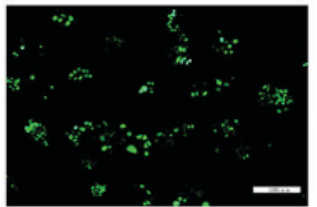

$\mathrm{H}_{2} \mathrm{O}_{2}$

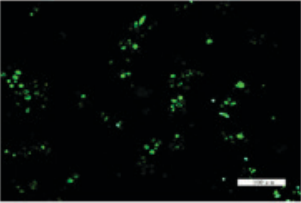

$\mathrm{H}_{2} \mathrm{O}_{2}+\mathrm{PEMF}$
B

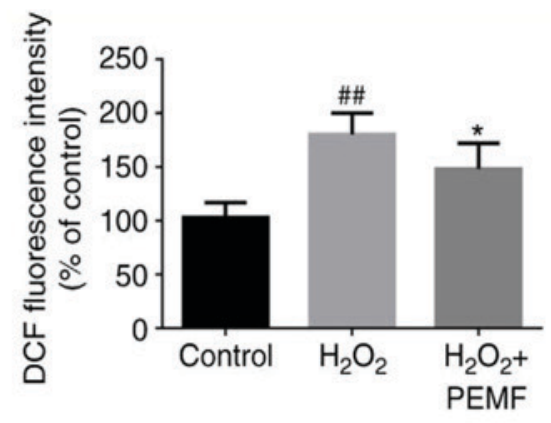

D

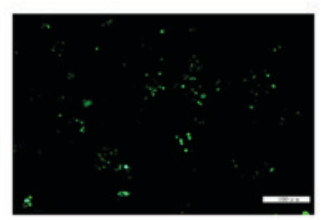

Control

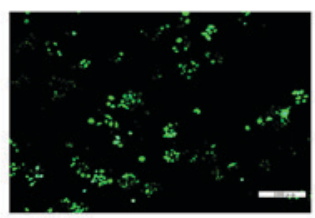

RANKL

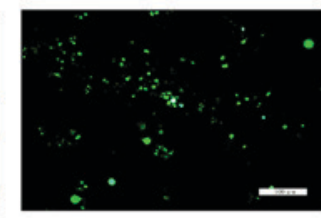

RANKL+PEMF

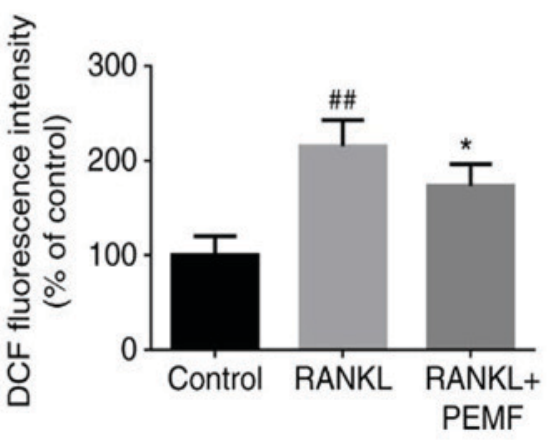

Figure 3. PEMF reduces the intracellular level of induced ROS. RAW264.7 cells were treated with $\mathrm{H}_{2} \mathrm{O}_{2}(1 \mathrm{mM})$ for 30 min or incubated with RANKL for 2 days in the absence and presence of PEMF. (A) Accumulation of intracellular ROS in $\mathrm{H}_{2} \mathrm{O}_{2}$-treated groups was assessed by fluorescence microscopy (scale bar $=100 \mu \mathrm{m}$ ) and (B) OD values of DCF in these groups were measured using a fluorescent reader at $\lambda=530 \mathrm{~nm}$. (C) Accumulation of intracellular ROS in the RANKL-treated groups was assessed by fluorescence microscopy (scale bar=100 $\mu \mathrm{m}$ ) and (D) OD values of DCF in these groups were measured using a fluorescent reader at $\lambda=530 \mathrm{~nm}$. All values are expressed as the mean \pm standard deviation $(\mathrm{n}=3)$. ${ }^{\# \#} \mathrm{P}<0.01$ compared with the control group; ${ }^{*} \mathrm{P}<0.05$ compared with $\mathrm{H}_{2} \mathrm{O}_{2}$ or RANKL alone. PEMF, pulsed electromagnetic field; RANKL, receptor activator of nuclear factor- $\mathrm{\kappa B}$ ligand; $\mathrm{H}_{2} \mathrm{O}_{2}$, hydrogen peroxide; ROS, reactive oxygen species; DCF, 2',7'-dichlorofluorescein; OD, optical density.

downregulated the expression of osteoclastogenesis-associated genes (Fig. 4D). These data revealed ROS to be the key factor in RANKL-induced osteoclast formation, and scavenging intracellular ROS effectively inhibited osteoclast formation.

\section{Discussion}

Osteoclasts are unique in their ability to resorb mineralized bone and have critical functions in bone remodeling and physical skeletal morphogenesis (28). In recent decades, it has been confirmed that electromagnetic fields, including PEMF, have potential in curing skeletal muscle system diseases and/or injury, including fracture healing, particularly in tendon-to-bone healing and joint and bone injury (29-31). Abnormalities in osteoclast number and activity are associated with osteoporosis. In the present study, it was evident that $75 \mathrm{~Hz}, 1 \mathrm{mt}$ PEMF exhibited a distinctly inhibitory effect on RANKL-induced osteoclast differentiation. Therefore, it is of clinical significance.

Previous studies have examined the action of PEMF in osteoporosis, attributing its mechanism of action to osteoblast precursor proliferation (32) and the inhibition of osteoclast activity (33), in addition to the enhancement of osteoblastic mineralization potential (9). Treatment of bone marrow cells derived from ovariectomized rats with PEMF led to significant suppression of osteoclast formation and osteoclast-associated cytokine expression (34). Furthermore, certain properties of deteriorated bone, including its stiffness, the maximum load and the yield load, are inhibited following PEMF exposure, indicating that more bone mass was retained and there was greater mechanical bone strength to resist fractures (35). Wang et al (36) also reported that RAW264.7 cells incubated with RANKL and exposed to $15 \mathrm{~Hz}$ PEMF ( $2 \mathrm{~h} /$ day) at $3 \mathrm{mt}$ for 7 days had decreased bone resorbing capacity, due to the promotion of osteoclast apoptosis. These results suggested that PEMF exerts biological effects on osteoclasts and/or osteoblasts. To better understand how PEMF affects osteoclasts, the present study also examined the effects of PEMF on osteoclast differentiation. Although it was demonstrated that PEMF inhibited RANKL-induced osteoclast differentiation by scavenging intracellular ROS, other possible mechanisms cannot be excluded. Another important regulating factor in osteoclasts is calcium. During RANKL-induced osteoclastogenesis, $\mathrm{Ca}^{2+}$ upregulates the downstream signal NFATc1, which is considered the master regulator of osteoclastogenesis (37). Previous studies have reported that PEMF affects the influx of $\mathrm{Ca}^{2+}$ in several cell types $(38,39)$. Therefore, $\mathrm{Ca}^{2+}$ may be another indication that PEMF affects osteoclast differentiation.

In the classic RANKL signaling pathway, the production of ROS is mediated by NADPH oxidase I in response to RANKL (40). In the present study, it was found that RANKL significantly increased intracellular ROS, consistent with previous reports. ROS are byproducts of cell metabolism and are also important signaling molecules. The ability of ROS to serve as signaling molecules in pathways has been well established, particularly in osteoclast differentiation, where ROS are 
A

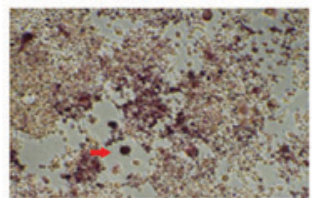

Control
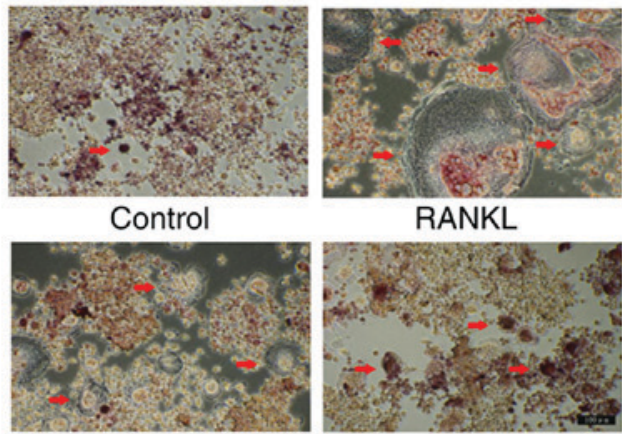

RANKL

RANKL+PEMF

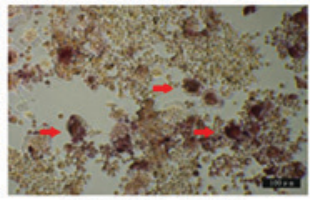

RANKL+DPI

B

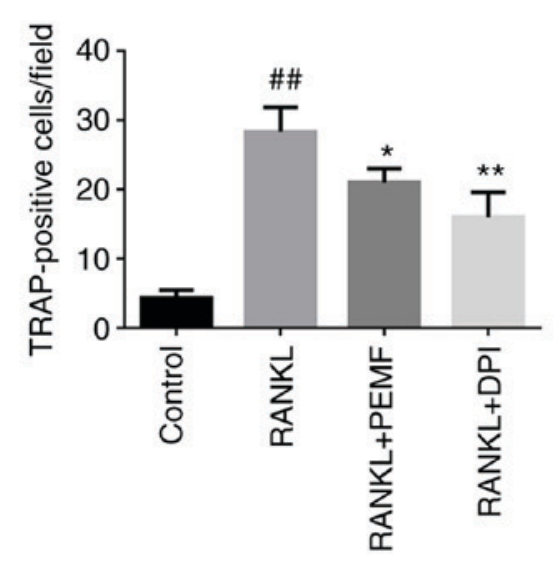

C

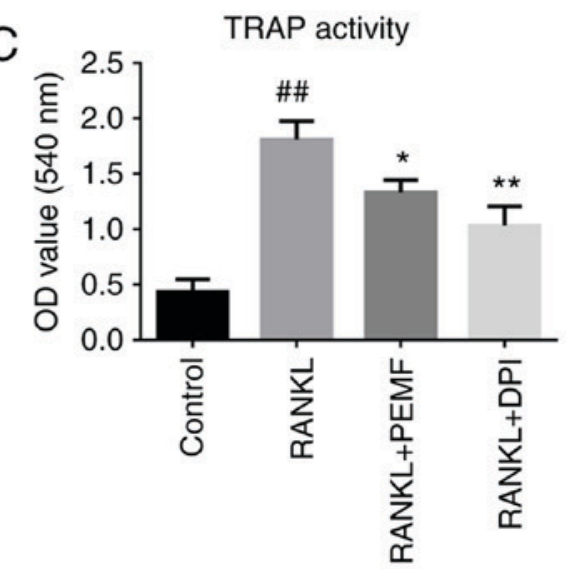

D
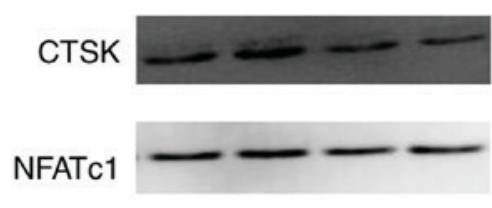

TRAP
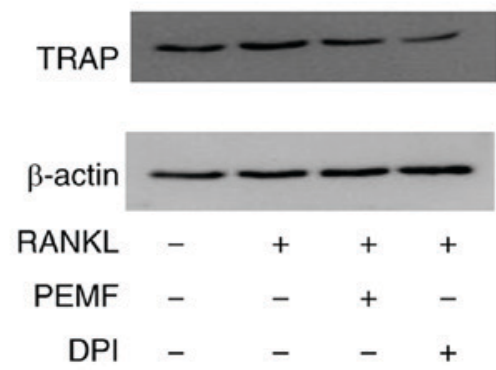

Figure 4. Reducing the level of intracellular ROS inhibits RANKL-induced osteoclast differentiation. RAW264.7 cells were treated with DPI (10 $\mu \mathrm{M})$ together with RANKL $(50 \mathrm{ng} / \mathrm{ml})$ for 4 days. (A) TRAP staining of osteoclasts treated with PEMF ( $3 \mathrm{~h} /$ day) or DPI together with RANKL for 4 days. Scale bar=100 $\mu \mathrm{m}$. (B) TRAP-positive multinucleated osteoclasts containing more than three nuclei were counted. (C) TRAP activity was measured at $\lambda=540 \mathrm{~nm}$ in each group. All values are expressed as the mean \pm standard deviation $(n=3)$. The relative expression level of the target gene was normalized to $\beta$-actin. ${ }^{\# \#} \mathrm{P}<0.01$ compared to the control group; " $\mathrm{P}<0.05,{ }^{* *} \mathrm{P}<0.01$ compared to RANKL alone. (D) Expression levels of osteoclastogenesis-associated proteins was analyzed. PEMF, pulsed electromagnetic field; RANKL, receptor activator of nuclear factor- $\mathrm{KB}$ ligand; DPI, diphenylene-iodonium chloride; TRAP, tartrate-resistant acid phosphatase; OD, optical density.

involved in the nuclear factor (NF)- $\mathrm{B}$ and mitogen-activated protein kinase (MAPK) signaling pathways (19). Additionally, the ROS-mediated direct modification of inositol 1,4,5-trisphosphate receptor thiol groups promotes $\mathrm{Ca}^{2+}$ release from the endoplasmic reticulum (41). As mentioned previously, $\mathrm{Ca}^{2+}$ is involved in osteoclast differentiation. Through NF-кB, MAPK and $\mathrm{Ca}^{2+}$ signaling, ROS are involved in the differentiation and formation of osteoclasts. DPI scavenges RANKL-induced intracellular ROS, effectively preventing the differentiation of osteoclast precursors. Furthermore, RANKL may upregulate the expression of superoxide dismutase 2 (SOD2) and sirtuin 3 (Sirt3), which exert feedback on osteoclastogenesis by inhibiting RANKL signaling via the downregulation of ROS (42). This mechanism physiologically prevents osteoclast overgrowth. Sirt3-deficient mice exhibit severe bone loss caused by elevated osteoclastogenesis (43). By contrast, evidence has revealed that several diseases are associated with ROS, including osteoporosis $(15,44)$. Certain antioxidants, including alliin and lycopene, inhibit osteoclast formation and bone resorption via the inhibition of ROS generation $(45,46)$. 
Exposure to PEMF leads to the upregulation of proteins associated with proper protein folding (47-50). A previous study revealed that PEMF effectively prevents the pro-oxidant effects of $\mathrm{H}_{2} \mathrm{O}_{2}$ in $\mathrm{SK}-\mathrm{N}-\mathrm{BE}(2)$ human neuroblastoma cells by increasing $\mathrm{Mn}$-dependent superoxide dismutase (MnSOD)-based antioxidant protection (51). Furthermore, Falone et al (52) found that PEMF markedly decreased ROS in human osteoclasts by inducing the expression of antioxidant enzymes, including glutathione peroxidase 3, SOD2, catalase and glutathione-s-reductase. However, whether the same mechanism exists in osteoclasts remains to be elucidated and requires further investigation.

There are certain limitations to the present study. First, the exact mechanism of PEMF scavenging of intracellular ROS in osteoclasts remains to be fully elucidated. As research in this area advances, there is no doubt that this issue can be addressed. Second, although RAW264.7 cells are widely used to induce the formation of osteoclasts in response to RANKL, they are not identical to osteoclasts derived from the body. For example, when comparing in vivo and in vitro conditions, there are discrepancies in vitamin D compound synthesis during osteoclastic bone resorption (53). Third, the present study did not determine the optimal PEMF parameters, including exposure time, strength and frequency, and did not perform bone resorption analysis or NOX expression assays. Therefore, further investigation is required.

In conclusion, the findings of the present study demonstrated that PEMF inhibited RANKL-induced osteoclast differentiation by scavenging intracellular ROS. These findings provide theoretical support for using PEMF in the treatment of osteoporosis and also indicates that ROS may be a potential therapeutic target in the treatment of osteoporosis.

\section{Acknowledgements}

The authors would like to thank Dr Ou Yang (Department of Human Anatomy, Basic Medical College, Southern Medical University) for the technical assistance of providing laboratory access.

\section{Funding}

This study was supported by the Science and Technology Planning Project of Guangdong Province, China (grant no. 2013B021800312).

\section{Availability of data and materials}

The datasets used and/or analyzed during the current study are available from the corresponding author on reasonable request.

\section{Authors' contributions}

JT conceived and designed the study. YP, HL, QY, YY, HX, YL and $\mathrm{ZH}$ performed the experiments. YP drafted the manuscript. HL, QY, YY, HX, YL and ZH reviewed and edited the manuscript. All authors read and approved the final manuscript.

\section{Ethics approval and consent to participate}

Not applicable.

\section{Patient consent for publication}

Not applicable.

\section{Competing interests}

The authors declare that they have no competing interests.

\section{References}

1. Lemaire V, Tobin FL, Greller LD, Cho CR and Suva LJ: Modeling the interactions between osteoblast and osteoclast activities in bone remodeling. J Theor Biol 229: 293-309, 2004.

2. Bloemen V, Schoenmaker T, de Vries TJ and Everts V: Direct cell-cell contact between periodontal ligament fibroblasts and osteoclast precursors synergistically increases the expression of genes related to osteoclastogenesis. J Cell Physiol 222: 565-573, 2010 .

3. Boyle WJ, Simonet WS and Lacey DL: Osteoclast differentiation and activation. Nature 423: 337-342, 2003.

4. Reid IR: Short-term and long-term effects of osteoporosis therapies. Nat Rev Endocrinol 11: 418-428, 2015.

5. Harvey N, Dennison E and Cooper C: Osteoporosis: Impact on health and economics. Nat Rev Rheumatol 6: 99-105, 2010.

6. Adami HO, Persson I, Hoover R, Schairer C and Bergkvist L: Risk of cancer in women receiving hormone replacement therapy. Int J Cancer 44: 833-839, 1989.

7. Lewiecki EM: Treatment of osteoporosis with denosumab. Maturitas 66: 182-186, 2010.

8. Jing D, Li F, Jiang M, Cai J, Wu Y, Xie K, Wu X, Tang C, Liu J, Guo W, et al: Pulsed electromagnetic fields improve bone microstructure and strength in ovariectomized rats through a Wnt/Lrp5/ $\beta$-catenin signaling-associated mechanism. PLoS One 8: e79377, 2013

9. Hannemann PF, Mommers EH, Schots JP, Brink PR and Poeze M: The effects of low-intensity pulsed ultrasound and pulsed electromagnetic fields bone growth stimulation in acute fractures: A systematic review and meta-analysis of randomized controlled trials. Arch Orthop Trauma Surg 134: 1093-1106, 2014.

10. Chang K, Chang WH, Tsai MT and Shih C: Pulsed electromagnetic fields accelerate apoptotic rate in osteoclasts. Connect Tissue Res 47: 222-228, 2006.

11. He Z, Selvamurugan N, Warshaw J and Partridge NC: Pulsed electromagnetic fields inhibit human osteoclast formation and gene expression via osteoblasts. Bone 106: 194-203, 2018.

12. Zhou J, Liao Y, Zeng Y, Xie H, Fu C and Li N: Effect of intervention initiation timing of pulsed electromagnetic field on ovariectomy-induced osteoporosis in rats. Bioelectromagnetics 38: 456-465, 2017.

13. Assiotis A, Sachinis NP and Chalidis BE: Pulsed electromagnetic fields for the treatment of tibial delayed unions and nonunions. A prospective clinical study and review of the literature. J Orthop Surg Res 7: 24, 2012.

14. Kim H, Kim IY, Lee SY and Jeong D: Bimodal actions of reactive oxygen species in the differentiation and bone-resorbing functions of osteoclasts. FEBS Lett 580: 5661-5665, 2006.

15. Domazetovic V, Marcucci G, Iantomasi T, Brandi ML and Vincenzini MT: Oxidative stress in bone remodeling: Role of antioxidants. Clin Cases Miner Bone Metab 14: 209-216, 2017.

16. Davies KJ: Oxidative stress: The paradox of aerobic life. Biochem Soc Symp 61: 1-31, 1995.

17. Forman HJ, Fukuto JM and Torres M: Redox signaling: Thiol chemistry defines which reactive oxygen and nitrogen species can act as second messengers. Am J Physiol Cell Physiol 287: C246-C256, 2004.

18. Lander HM: An essential role for free radicals and derived species in signal transduction. FASEB J 11: 118-124, 1997.

19. Callaway DA and Jiang JX: Reactive oxygen species and oxidative stress in osteoclastogenesis, skeletal aging and bone diseases. J Bone Miner Metab 33: 359-370, 2015. 
20. Lean JM, Davies JT, Fuller K, Jagger CJ, Kirstein B, Partington GA, Urry ZL and Chambers TJ: A crucial role for thiol antioxidants in estrogen-deficiency bone loss. J Clin Invest 112: 915-923, 2003.

21. Moon HJ, Ko WK, Han SW, Kim DS, Hwang YS, Park HK and Kwon IK: Antioxidants, like coenzyme Q10, selenite, and curcumin, inhibited osteoclast differentiation by suppressing reactive oxygen species generation. Biochem Biophys Res Commun 418: 247-253, 2012

22. Moon HJ, Kim SE, Yun YP, Hwang YS, Bang JB, Park JH and Kwon IK: Simvastatin inhibits osteoclast differentiation by scavenging reactive oxygen species. Exp Mol Med 43: 605-612, 2011

23. Garrett IR, Boyce BF, Oreffo RO, Bonewald L, Poser J and Mundy GR: Oxygen-derived free radicals stimulate osteoclastic bone resorption in rodent bone in vitro and in vivo. J Clin Invest 85: 632-639, 1990.

24. Ishii KA, Fumoto T, Iwai K, Takeshita S, Ito M, Shimohata N, Aburatani H, Taketani S, Lelliott CJ, Vidal-Puig A and Ikeda K: Coordination of PGC-1beta and iron uptake in mitochondrial biogenesis and osteoclast activation. Nat Med 15: 259-266, 2009

25. Bartell SM, Kim HN, Ambrogini E, Han L, Iyer S, Serra Ucer S, Rabinovitch P, Jilka RL, Weinstein RS, Zhao H, et al: FoxO proteins restrain osteoclastogenesis and bone resorption by attenuating $\mathrm{H}_{2} \mathrm{O}_{2}$ accumulation. Nat Commun 5: 3773, 2014.

26. Livak KJ and Schmittgen TD: Analysis of relative gene expression data using real-time quantitative PCR and the 2(-Delta Delta C(T)) method. Methods 25: 402-408, 2001.

27. Lee SH, Ding Y, Yan XT, Kim YH and Jang HD: Scopoletin and scopolin isolated from Artemisia iwayomogi suppress differentiation of osteoclastic macrophage RAW 264.7 cells by scavenging reactive oxygen species. J Nat Prod 76: 615-620, 2013.

28. Teitelbaum SL and Ross FP: Genetic regulation of osteoclast development and function. Nat Rev Genet 4: 638-649, 2003.

29. Tucker JJ, Cirone JM, Morris TR, Nuss CA, Huegel J, Waldorff EI, Zhang N, Ryaby JT and Soslowsky LJ: Pulsed electromagnetic field therapy improves tendon-to-bone healing in a rat rotator cuff repair model. J Orthop Res 35: 902-909, 2017.

30. Osti L, Buono AD and Maffulli N: Pulsed electromagnetic fields after rotator cuff repair: A randomized, controlled study. Orthopedics 38: e223-e228, 2015.

31. Veronesi F, Torricelli P, Giavaresi G, Sartori M, Cavani F, Setti S, Cadossi M, Ongaro A and Fini M: In vivo effect of two different pulsed electromagnetic field frequencies on osteoarthritis. J Orthop Res 32: 677-685, 2014.

32. Hartig M, Joos U and Wiesmann HP: Capacitively coupled electric fields accelerate proliferation of osteoblast-like primary cells and increase bone extracellular matrix formation in vitro. Eur Biophys J 29: 499-506, 2000.

33. Lohmann CH, Schwartz Z, Liu Y, Guerkov H, Dean DD, Simon B and Boyan BD: Pulsed electromagnetic field stimulation of MG63 osteoblast-like cells affects differentiation and local factor production. J Orthop Res 18: 637-646, 2000.

34. He J, Zhang Y, Chen J, Zheng S, Huang H and Dong X: Effects of pulsed electromagnetic fields on the expression of NFATc1 and CAII in mouse osteoclast-like cells. Aging Clin Exp Res 27: 13-19, 2015.

35. Jing D, Cai J, Wu Y, Shen G, Li F, Xu Q, Xie K, Tang C, Liu J, Guo W, et al: Pulsed electromagnetic fields partially preserve bone mass, microarchitecture, and strength by promoting bone formation in hindlimb-suspended rats. J Bone Miner Res 29: 2250-2261, 2014.

36. Wang P, Liu J, Yang Y, Zhai M, Shao X, Yan Z, Zhang X, Wu Y, Cao L, Sui B, et al: Differential intensity-dependent effects of pulsed electromagnetic fields on RANKL-induced osteoclast formation, apoptosis, and bone resorbing ability in RAW264.7 cells. Bioelectromagnetics 38: 602-612, 2017.

37. Negishi-Koga T and Takayanagi $\mathrm{H}: \mathrm{Ca}^{2+}-\mathrm{NFATc1}$ signaling is an essential axis of osteoclast differentiation. Immunol Rev 231: 241-256, 2009
38. Pall ML: Electromagnetic fields act via activation of voltage-gated calcium channels to produce beneficial or adverse effects. J Cell Mol Med 17: 958-965, 2013

39. Cornacchione M, Pellegrini M, Fassina L, Mognaschi ME, Di Siena S, Gimmelli R, Ambrosino P, Soldovieri MV, Taglialatela M, Gianfrilli $\mathrm{D}$, et al: $\beta$-Adrenergic response is counteracted by extremely-low-frequency pulsed electromagnetic fields in beating cardiomyocytes. J Mol Cell Cardiol 98: 146-158, 2016.

40. Lee NK, Choi YG, Baik JY, Han SY, Jeong DW, Bae YS, Kim N and Lee SY: A crucial role for reactive oxygen species in RANKL-induced osteoclast differentiation. Blood 106: 852-859, 2005.

41. Decuypere JP, Monaco G, Missiaen L, De Smedt H, Parys JB and Bultynck G: IP(3) receptors, mitochondria, and Ca signaling: Implications for aging. J Aging Res 2011: 920178, 2011.

42. Kim H, Lee YD, Kim HJ, Lee ZH and Kim HH: SOD2 and Sirt3 control osteoclastogenesis by regulating mitochondrial ROS. J Bone Miner Res 32: 397-406, 2017.

43. Huh JE, Shin JH, Jang ES, Park SJ, Park DR, Ko R, Seo DH, Kim HS, Lee SH, Choi Y, et al: Sirtuin 3 (SIRT3) maintains bone homeostasis by regulating AMPK-PGC-1 $\beta$ axis in mice. Sci Rep 6: 22511, 2016.

44. Sendur OF, Turan Y, Tastaban E and Serter M: Antioxidant status in patients with osteoporosis: A controlled study. Joint Bone Spine 76: 514-518, 2009

45. Chen Y, Sun J, Dou C, Li N, Kang F, Wang Y, Cao Z, Yang X and Dong S: Alliin attenuated RANKL-induced osteoclastogenesis by scavenging reactive oxygen species through inhibiting Nox1. Int J Mol Sci 17: pii: E1516, 2016.

46. Rao LG, Krishnadev N, Banasikowska K and Rao AV: Lycopene I-effect on osteoclasts: Lycopene inhibits basal and parathyroid hormone-stimulated osteoclast formation and mineral resorption mediated by reactive oxygen species in rat bone marrow cultures. J Med Food 6: 69-78, 2003

47. Falone S, Grossi MR, Cinque B, D'Angelo B, Tettamanti E, Cimini A, Di Ilio C and Amicarelli F: Fifty hertz extremely low-frequency electromagnetic field causes changes in redox and differentiative status in neuroblastoma cells. Int J Biochem Cell Biol 39: 2093-2106, 2007.

48. Simkó M: Cell type specific redox status is responsible for diverse electromagnetic field effects. Curr Med Chem 14: 1141-1152, 2007.

49. Mannerling AC, Simko M, Mild KH and Mattsson MO: Effects of $50-\mathrm{Hz}$ magnetic field exposure on superoxide radical anion formation and HSP70 induction in human K562 cells. Radiat Environ Biophys 49: 731-741, 2010.

50. Osera C, Fassina L, Amadio M, Venturini L, Buoso E, Magenes G, Govoni S, Ricevuti G and Pascale A: Cytoprotective response induced by electromagnetic stimulation on SH-SY5Y human neuroblastoma cell line. Tissue Eng Part A 17: 2573-2582, 2011.

51. Ehnert S, Fentz AK, Schreiner A, Birk J, Wilbrand B, Ziegler P, Reumann MK, Wang H, Falldorf K and Nussler AK: Extremely low frequency pulsed electromagnetic fields cause antioxidative defense mechanisms in human osteoblasts via induction of $\cdot \mathrm{O}_{2}$ and $\mathrm{H}_{2} \mathrm{O}_{2}$. Sci Rep 7: 14544, 2017.

52. Falone S, Marchesi N, Osera C, Fassina L, Comincini S, Amadio $\mathrm{M}$ and Pascale A: Pulsed electromagnetic field (PEMF) prevents pro-oxidant effects of $\mathrm{H}_{2} \mathrm{O}_{2}$ in SK-N-BE(2) human neuroblastoma cells. Int J Radiat Biol 92: 281-286, 2016.

53. Suda T, Takahashi $F$ and Takahashi N: Bone effects of vitamin D-discrepancies between in vivo and in vitro studies. Arch Biochem Biophys 523: 22-29, 2012.

This work is licensed under a Creative Commons Attribution-NonCommercial-NoDerivatives 4.0 International (CC BY-NC-ND 4.0) License. 